\title{
Longitudinal diffusion tensor imaging in amyotrophic lateral sclerosis
}

\author{
Carsten Keil ${ }^{3+}$, Tino Prell ${ }^{1 * \dagger}$, Thomas Peschel ${ }^{2}$, Viktor Hartung ${ }^{1}$, Reinhard Dengler ${ }^{3}$ and Julian Grosskreutz ${ }^{1}$
}

\begin{abstract}
Background: Amyotrophic lateral sclerosis (ALS) is a fatal neurodegenerative disorder, caused by progressive loss of motor neurons. Changes are widespread in the subcortical white matter in ALS. Diffusion tensor imaging (DTI) detects pathological changes in white matter fibres in vivo, based on alterations in the degree (diffusivity, ADC) and directedness (fractional anisotropy, FA) of proton movement.

Methods: 24 patients with ALS and 24 age-matched controls received 1.5T DTI. FA and ADC were analyzed using statistical parametric mapping. In 15 of the 24 ALS patients, a second DTI was obtained after 6 months.

Results: Decreased FA in the corticospinal tract (CST) and frontal areas confirm existing results. With a direct comparison of baseline and follow-up dataset, the progression of upper motor neuron degeneration, reflected in FA decrease, could be captured along the CST and in frontal areas. The involvement of cerebellum in the pathology of ALS, as suspected from functional MRI studies, could be confirmed by a reduced FA (culmen, declive). These structural changes correlated well with disease duration, ALSFRS-R, and physical and executive functions.

Conclusion: DTI detects changes that are regarded as prominent features of ALS and thus, shows promise in its function as a biomarker. Using the technique herein, we could demonstrate DTI changes at follow-up which correlated well with clinical progression.
\end{abstract}

Keywords: Cerebellum, Amyotrophic lateral sclerosis, Diffusion tensor imaging, Follow-up

\section{Background}

Amyotrophic lateral sclerosis (ALS) is a neurodegenerative disorder characterized by progressive failure of upper and lower motor neurons. In the ALS brain, degeneration of fibres is widespread in the subcortical white matter with surrounding astrogliosis. Degeneration commonly extends into the corticospinal tract (CST), the central part of the corpus callosum and in frontal areas as well as in long projection fibres as seen in post-mortem tissue [1,2]. Previous studies have demonstrated white matter changes in ALS using diffusion tensor imaging (DTI), a technique that detects alterations in both degree (diffusivity, ADC) and directedness (fractional anisotropy, FA) of proton movement reflecting changes in microstructural tissue and fibre organisation [3]. In ALS, DTI revealed a variable, but

\footnotetext{
* Correspondence: tino.prell@med.uni-jena.de

${ }^{\dagger}$ Equal contributors

'Department of Neurology, Jena University Hospital, Erlanger Allee 101, Jena 07747, Germany

Full list of author information is available at the end of the article
}

widespread pattern of white matter abnormalities which were most prominent along the CST and the subcentral white matter, and which extended into frontal regions [4-9].

Symptoms indicating involvment of lower motor neurons such as atrophic paresis may sometimes obscure signs of upper motor neuron failure leading to a delay in diagnosis and treatmentof ALS. Therefore, a biomarker for diagnostic, prognostic, and monitoring purposes is needed. Here, DTI shows promise as a potential biomarker, however, longitudinal studies are required to further clarify the time course and distribution of changesdetected by this technique. By customizing scripting in the Statistical Parametric Mapping software (SPM, http://www.fil.ion.ucl.ac.uk/spm) we aimed to analyze DTI datasets from 24 ALS patients, without having to make a priori assumptions about localization of pathology. To obtain longitudinal data on progress of microstructural changes in ALS, a second examination was undertaken in a subset of 15 patients at 6 months.

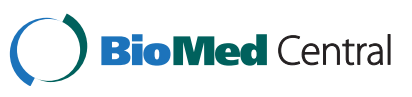




\section{Methods}

\section{Subjects}

We studied DTI image sets of 24 patients (T0) with sporadic ALS and of 24 healthy age-matched controls. In a subset of 15 of the 24 ALS patients (Follow-up), a second examination was performed 6 months later. In the 24 ALS patients, the mean ( \pm S.D.) duration since onset of dysarthria or weakness (termed disease duration) at MRI was $25.6( \pm 27,8)$ months, the mean age was $62.4 \pm 10.5$ (range 32.7-75.9) years, and the mean revised ALS functional rating scale (ALSFRS-R) was $36.3 \pm 9$ points. The female to male ratio was 1:1. 9 patients had a bulbar onset and 15 patients had, limb onset of weakness. All patients were being treated with riluzole and none were taking psychoactive drugs. Significant frontal or cognitive dysfunctions were ruled out by means of the Mini Mental State Examination (MMSE) and the Frontal Assessment Battery test (FAB). In addition, the 36-item Short Form Health Survey (SF36) was completed by all patients enrolled and the physical and executive subset was used for correlation analysis. The 24 age-matched healthy controls (mean age $61.6 \pm$ 9.2, range $36.6-71.9$ ) had no history of a disease of the central nervous system, their neurological examinations were normal, and MRI T1 and T2 images revealed no pathological findings. Finally, clinical diagnosis of ALS was made according to the revised El-Escorial criteria. Disease characteristics of patients enrolled in the study are summarized in Table 1 [10]. All subjects gave written informed consent to participate in the study, which was approved by the local ethics committee.

\section{Data acquisition}

Images were acquired on a neuro-optimized 1.5-T GE Signa Horizon LX (General Electric Company, Milwaukee, WI, USA) using a 3-dimensional T1-weighted spoiled gradient recalled echo (SPGR) sequence generating 124 contiguous sagittal slices (RT 24ms; TE $8 \mathrm{~ms}$; flip angle

Table 1 Characteristics of ALS patients

\begin{tabular}{|c|c|c|c|c|c|c|c|c|c|c|c|c|c|}
\hline \multirow[t]{2}{*}{$\#$} & \multirow[t]{2}{*}{ Age } & \multirow[t]{2}{*}{ Sex } & \multirow[t]{2}{*}{ Onset } & \multirow[t]{2}{*}{ El escorial } & \multirow{2}{*}{$\begin{array}{c}\text { Disease duration } \\
\text { mth }\end{array}$} & \multicolumn{2}{|c|}{ MMST } & \multicolumn{2}{|c|}{ FAB } & \multicolumn{2}{|c|}{ ALSFRS-R } & \multicolumn{2}{|c|}{ SF36 physic } \\
\hline & & & & & & 0 & 6 & 0 & 6 & 0 & 6 & 0 & 6 \\
\hline $1^{*}$ & 67.9 & $f$ & $b$ & $p$ & 3 & 30 & & 17 & & 45 & 45 & 100 & 100 \\
\hline 2 & 72.5 & $f$ & $b$ & $p$ & 6 & 30 & 30 & 17 & 18 & 44 & & 100 & \\
\hline $3^{*}$ & 58.1 & $f$ & $b$ & $p$ & 4 & 29 & 28 & 16 & 18 & 46 & 43 & 25 & 20 \\
\hline $4^{*}$ & 72.0 & $m$ & 1 & $p$ & 31 & 30 & 29 & 17 & 17 & 39 & 32 & 35 & 35 \\
\hline 5 & 75,9 & $m$ & 1 & $p$ & 15 & 29 & & 12 & & 25 & & 5 & \\
\hline $6^{*}$ & 64,8 & $\mathrm{~m}$ & 1 & $d$ & 33 & 29 & 28 & 15 & 12 & 32 & 27 & 0 & 0 \\
\hline 7 & 64,4 & $m$ & 1 & $p$ & 32 & 30 & & 16 & & 43 & & 20 & \\
\hline $8^{*}$ & 32,7 & $m$ & 1 & $d$ & 19 & 30 & 30 & 18 & 18 & 30 & 27 & 0 & 0 \\
\hline $9^{*}$ & 44,3 & $f$ & 1 & $d$ & 32 & 29 & no & 18 & no & 18 & 18 & 0 & 0 \\
\hline $10^{*}$ & 64,5 & $f$ & 1 & $\mathrm{~s}$ & 3 & 28 & 27 & 15 & 9 & 45 & 35 & 10 & 5 \\
\hline $11^{*}$ & 69,3 & $m$ & $b$ & $d$ & 16 & 30 & 29 & 14 & 16 & 33 & 32 & 0 & 0 \\
\hline $12^{*}$ & 70 & $f$ & 1 & $\mathrm{~s}$ & 37 & 27 & 30 & 15 & 18 & 35 & 31 & 10 & 10 \\
\hline 13 & 68,6 & $f$ & $b$ & $p$ & 7 & 21 & & 12 & & 47 & & 100 & \\
\hline $14^{*}$ & 67,5 & $\mathrm{~m}$ & 1 & $p$ & 22 & 29 & 28 & 18 & 18 & 45 & 45 & 95 & 95 \\
\hline $15^{*}$ & 49,7 & $\mathrm{~m}$ & 1 & $\mathrm{~s}$ & 10 & 30 & & 17 & & 47 & & 60 & 0 \\
\hline $16^{*}$ & 64,8 & $f$ & 1 & $d$ & 52 & 30 & 30 & 18 & 18 & 37 & 37 & 0 & 0 \\
\hline 17 & 48,3 & $f$ & 1 & $d$ & 104 & no & no & no & no & 21 & 21 & 0 & \\
\hline $18^{*}$ & 71,3 & $\mathrm{~m}$ & $b$ & $p$ & 8 & 27 & & 15 & & 40 & & 35 & 15 \\
\hline 19 & 60,2 & $\mathrm{~m}$ & 1 & $p$ & 18 & 29 & 30 & 15 & 18 & 39 & 39 & 25 & \\
\hline 20 & 54,9 & $d$ & 1 & $p$ & 13 & 29 & & 18 & & 27 & & 0 & \\
\hline $21^{*}$ & 55,1 & $m$ & $b$ & $p$ & 2 & 29 & & 16 & & 45 & & 85 & 0 \\
\hline $22^{*}$ & 64,1 & $f$ & $b$ & $d$ & 13 & 30 & 30 & 15 & 15 & 34 & 25 & 45 & 30 \\
\hline 23 & 74,4 & $\mathrm{~m}$ & 1 & $p$ & 107 & 30 & 30 & 17 & 17 & 32 & 32 & 30 & \\
\hline $24^{*}$ & 65,3 & $f$ & $b$ & $p$ & 28 & 29 & & 18 & & 21 & & 0 & 15 \\
\hline $\mathrm{MW}$ & 61,5 & & & & 25,6 & 28,9 & 29,2 & 16,0 & 16,3 & 36,3 & 32,6 & 32,5 & 20,3 \\
\hline SD & 10,9 & & & & 27,8 & 1,9 & 1,1 & 1,8 & 2,8 & 9,0 & 8,3 & 37,2 & 32,2 \\
\hline
\end{tabular}

S.D. standard deviation, $\mathrm{f} / \mathrm{m}$ female/male ratio, s suspected, p probable, d definite, I limb onset, b bulbar onset, T0 (month 0), 6 follow-up (month 6), * patients, who underwent follow-up. 
$30^{\circ}, 2$ averages, acquisition time 13'10", in plane resolution $0.97 \times 0.97 \times 1.5 \mathrm{~mm}$ [3]). DTI was performed using echoplanar imaging (EPI) (39 contiguous slices, $3 \mathrm{~mm}$ thickness, $2 \times 2 \mathrm{~mm}$ in plane resolution, 24 directions, $b=1000$, total scanning time $25 \mathrm{~min}$ ). During scanning, all participants were placed comfortably and their heads were fixated within the headcoil using special cushions. All subjects received additional T2-weighted images to exclude cerebrovascular disease.

\section{Pre-processing}

Data were processed on a standard IBM-compatible PC using SPM2 statistical parametric mapping software (Welcome Department of Cognitive Neurology, London) in the analysis environment MATLAB (version 6.1; the Math Works Inc, Natick, Mass). Images were reoriented into oblique axial slices aligned parallel to the anteriorposterior commissural axis with the origin set to the anterior commissure.

After calculatingthe FA and ADC maps, images were pre-processed and analyzed by statistical parametric mapping (SPM2) using an approach adopted from voxel-based morphometry as described herein by our group [11]. This included an optimized normalisation procedure, together with an automated exclusion of skull and CSF signal values and smoothing (6-mm FWHM). All EPI scans were normalized to the EPItemplate provided by SPM and further used to create a site-specific EPI template appropriate to the population sample and with scanner specific image contrast. This site-specifc template was used again for normalisation and brain extraction for the individual images in the group studied, which resulted in optimal normalization and cleaning parameters for use with the FA and ADC images.

\section{Statistical analysis}

Processed images of each tissue class were analyzed in the framework of the general linear model. ANCOVA was applied to compare the groups of patients and healthy controls ( $\mathrm{p}<0.05$, corrected for the entire volume). Additionally, regression analyses with clinical measures were explored for ALS patients using the SPM2 model 'single subjects: covariates only'. In accordance with our anatomical a priori hypothesis regarding white matter changes in ALS along the CST, the statistical threshold was set at $\mathrm{p}<0.001$, corrected. For regions where changes could be expected in the follow-up (CST) [11,12], a small volume correction (SVC) was undertaken and the family-wise-error (FWE)-method $(\mathrm{P}<0,05)$ for multiple comparisons was used.

\section{Results}

\section{Clinical characterization}

A total of 24 patients with sporadic ALS and the same number of age- and gender-matched healthy controls underwent MRI (T0) . In addition, DTI was performed in 15 patients with ALS after six months (follow-up). Based on MMST and FAB, no significant cognitive or executive impairment was observed in the T0 (MMST $28.8 \pm 1.57$, FAB $16.04 \pm 1.80)$ and in the follow-up group (MMST $29.2 \pm 1.07$, FAB $16.31 \pm 2.81$ ). Three patients switched from possible to probable laboratorysupported ALS according to the revised El Escorial criteria. In the follow-up group, theALSFRS-R and the physical functioning SF36 subscore decreased from 35.4 $( \pm 8.4)$ to $32,6( \pm 8.3)$ and from $25,0( \pm 33,0)$ to 20,7 $( \pm 33,3)$ points, respectively after 6 months. Both reductions were not significant. Clinical characteristics of patients are summarized in Table 1.

\section{Whole-brain group differences in FA and ADC at T0}

In the motor system, the FA in the ALS patient group $(n=24)$ was reduced within the precentral gyrus, in the supplementary motor cortex, as well as in the CST at the level of corona radiata, pons, and crus cerebri compared to healthy controls. Further, FA was reduced in the corpus callosum, the parahippocampal gyrus and in frontal areas (Table 2).

Diffusivity was enhanced in the motor system (precentral gyrus, premotor area, CST at the level of corona radiate, pons), the postcentral gyrus, and in frontal and temporal areas (Table 2). Enhanced diffusivity in the pons was rostrally located to pontine FA decrease.

\section{Whole-brain group differences in FA and ADC at follow-up} According to T0 MRI in ALS patients $(n=15)$ after 6 months, the FA was reduced in the CST (corona radiate), the pons, the corpus callosum, and the parahippocampal gyrus. Additionally, FA was found decreased in frontal areas (gyrus frontalis medialis), insula, in the limbic system (amygdale), and in the thalamus at this time point (Table 2, Figure 1).

In the follow-up group, the diffusivity was again enhanced in the motor system (right precentral gyrus, and in the CST at the level of corona radiate, internal capsula). After SVC, there was a decrease in FA bilaterally in the WM of precentral gyrus, along the CST (corona radiate), and in the limbic system (fusiform gyrus, uncus) (Table 2, Figure 1). After 6 months, microstructural changes had progressed along the descending fibres and into the internal capsule.

\section{Comparison of T0 to follow-up}

Results of the 15 ALS patients, who had undergone follow-up DTI analysis were compared to their T0 data 
Table 2 Results of group comparisons of ALS patients versus controls

\begin{tabular}{|c|c|c|c|c|c|c|c|}
\hline & Side & Cluster size & $\mathbf{x}$ & y & $z$ & $T$ & $\mathrm{p}$ \\
\hline \multicolumn{8}{|l|}{ T0 FA all ALS patients $(n=24)<$ controls } \\
\hline Corpus Callosum & I & 7954 & -10 & -14 & 29 & 6.03 & 0.002 \\
\hline Corpus Callosum & । & & -1 & -16 & 25 & 5.35 & 0.003 \\
\hline Corpus Callosum & $r$ & & 11 & -16 & 29 & 5.29 & 0.003 \\
\hline Brainstem, Pons, CST & I & 1589 & -8 & -27 & -34 & 5.82 & 0.002 \\
\hline Brainstem, Pons, CST & $r$ & & 9 & -26 & -36 & 4.54 & 0.007 \\
\hline Brainstem, Pons, CST & $r$ & & 10 & -22 & -24 & 3.88 & 0.027 \\
\hline Precentral Gyrus, WM & r & 2386 & 14 & -21 & 58 & 5.69 & 0.002 \\
\hline subgyral, WM, Corona Radiata & r & & 27 & -38 & 50 & 4.02 & 0.021 \\
\hline Medial Frontal Gyrus, WM, below BA 6 & I & 743 & -12 & -13 & 70 & 5.03 & 0.003 \\
\hline Precentral Gyrus, WM & $r$ & 290 & 48 & 4 & 8 & 4.59 & 0.007 \\
\hline Corona Radiata, Medial Frontal Gyrus, below BA 6 & 1 & 687 & -35 & -12 & 48 & 4.37 & 0.01 \\
\hline Precentral Gyrus, WM & I & & -39 & -19 & 48 & 4.18 & 0.015 \\
\hline subgyral, WM, below Gyrus postcentralis & I & 201 & -10 & -37 & 61 & 4.35 & 0.011 \\
\hline Precentral Gyrus, subgyral, WM, Corona Radiata & I & 343 & -42 & -15 & 23 & 4.34 & 0.011 \\
\hline Precentral Gyrus, WM & 1 & 942 & -28 & -24 & 55 & 4.26 & 0.013 \\
\hline Frontal Lobe, subgyral, WM, Corona radiata & 1 & & -17 & -24 & 55 & 3.62 & 0.042 \\
\hline Frontal Lobe, subgyral, WM, Corona radiata & $r$ & 345 & 33 & -8 & 38 & 3.87 & 0.028 \\
\hline Frontal Lobe, Precentral Gyrus, WM, Corona radiata & 1 & 309 & -39 & -6 & 35 & 3.86 & 0.028 \\
\hline Frontal Lobe, Precentral Gyrus, BA 6 & 1 & & -46 & 1 & 40 & 3.82 & 0.03 \\
\hline Limbic Lobe, Parahippocampal Gyrus, WM & I & 218 & -20 & -48 & -11 & 3.80 & 0.032 \\
\hline \multicolumn{8}{|l|}{ T0 ADC all ALS patients $(n=24)>$ controls } \\
\hline Precentral Gyrus, WM & 1 & 5108 & -9 & -25 & 67 & 5.99 & 0.02 \\
\hline Precentral Gyrus, subgyral, WM, below BA 6 & I & & -28 & -16 & 65 & 5.5 & 0.02 \\
\hline Frontal Lobe, subgyral, WM, Corona radiata & 1 & & -28 & -25 & 50 & 5.33 & 0.02 \\
\hline Precentral Gyrus, subgyral, WM & $r$ & 5537 & 21 & -16 & 65 & 5.56 & 0.02 \\
\hline Precentral Gyrus, WM & $r$ & & 11 & -23 & 67 & 5.22 & 0.02 \\
\hline Frontal Lobe, subgyral, WM, Postcentral Gyrus & r & & 15 & -34 & 61 & 4.31 & 0.031 \\
\hline Brainstem, Pons & $r$ & 3243 & 6 & -22 & -28 & 5.13 & 0.02 \\
\hline Brainstem, Pons & I & & -4 & -23 & -28 & 4.21 & 0.034 \\
\hline Limbic Lobe, Parahippocampal Gyrus, WM & r & & 26 & -14 & -14 & 3.98 & 0.041 \\
\hline Temporal Lobe, Superior Temporal Gyrus, GM & 1 & 547 & -62 & -12 & -1 & 4.90 & 0.02 \\
\hline Parietal Lobe, Superior Parietal Lobule, subgyral, BA 7 & r & 754 & 21 & -50 & 65 & 4.73 & 0.022 \\
\hline Precentral Gyrus, WM & r & 336 & 47 & -2 & 31 & 4.55 & 0.025 \\
\hline Postcentral Gyrus, subgyral, WM & 1 & 645 & -18 & -32 & 71 & 4.22 & 0.034 \\
\hline Postcentral Gyrus, subgyral, WM & 1 & & -27 & -31 & 67 & 4.02 & 0.04 \\
\hline Frontal Lobe, Superior Frontal Gyrus, GM, BA 9 & I & 467 & -22 & 42 & 39 & 4.15 & 0.036 \\
\hline Frontal Lobe, subgyral, WM & I & & -25 & 39 & 29 & 3.78 & 0.046 \\
\hline Temporal Lobe, subgyral, WM & 1 & 409 & -32 & 9 & -35 & 4.11 & 0.037 \\
\hline Frontal Lobe, Superior Frontal Gyrus, WM & r & 269 & 5 & 20 & 53 & 4.02 & 0.04 \\
\hline Frontal Lobe, Medial Frontal Gyrus, GM, BA 8 & 1 & 383 & -6 & 27 & 53 & 4.02 & 0.04 \\
\hline Limbic Lobe, Uncus, WM & r & 827 & 22 & -3 & -36 & 3.95 & 0.041 \\
\hline Limbic Lobe & r & & 22 & -8 & -43 & 3.68 & 0.049 \\
\hline Frontal Lobe, Precentral Gyrus, subgyral, WM & 1 & 231 & -51 & -3 & 23 & 3.91 & 0.042 \\
\hline Sub-lobar, Insula, WM & I & 208 & -40 & -17 & 19 & 3.89 & 0.043 \\
\hline Frontal Lobe, Superior Frontal Gyrus, GM & । & 233 & -22 & 10 & 57 & 3.88 & 0.043 \\
\hline Frontal Lobe, Middle Frontal Gyrus, GM, BA 8 & I & 217 & -23 & 20 & 50 & 3.87 & 0.043 \\
\hline
\end{tabular}


Table 2 Results of group comparisons of ALS patients versus controls (Continued)

\begin{tabular}{|c|c|c|c|c|c|c|c|}
\hline Frontal Lobe, Middle Frontal Gyrus, GM, BA 10 & r & 365 & 27 & 53 & 26 & 3.81 & 0.045 \\
\hline Frontal Lobe, Superior Frontal Gyrus, GM, BA 9 & r & & 24 & 45 & 39 & 3.71 & 0.048 \\
\hline \multicolumn{8}{|l|}{ Follow-up FA all ALS patients $(n=15)<$ controls } \\
\hline Precentral Gyrus, subgyral, WM, Corona radiata & r & 293 & 48 & 4 & 6 & 6.26 & 0.017 \\
\hline Corpus Callosum & I & 3260 & -8 & -18 & 28 & 5.70 & 0.017 \\
\hline Corpus Callosum & $r$ & & 11 & -17 & 28 & 4.8 & 0.026 \\
\hline Left Brainstem, Pons & I & 218 & -6 & -28 & -38 & 5.02 & 0.02 \\
\hline Limbic Lobe, Parahippocampal Gyrus, WM & I & 1004 & -22 & -42 & -10 & 4.89 & 0.023 \\
\hline Temporal Lobe, Fusiform Gyrus, GM, BA 37 & I & & -27 & -37 & -16 & 4.26 & 0.04 \\
\hline Sub-lobar, Thalamus, GM, Pulvinar & 1 & 217 & -13 & -33 & 1 & 4.51 & 0.033 \\
\hline Frontal Lobe, Medial Frontal Gyrus, WM, Corona radiata & r & 696 & 13 & -26 & 58 & 4.44 & 0.035 \\
\hline Frontal Lobe, Medial Frontal Gyrus, WM, Corona radiata & r & & 9 & -27 & 69 & 4.28 & 0.04 \\
\hline parahippocampal Amygdala & r & 206 & 29 & -30 & -26 & 4.36 & 0.037 \\
\hline Limbic Lobe, Parahippocampal Gyrus, GM, BA 34 & r & 204 & 17 & -7 & -19 & 4.20 & 0.043 \\
\hline \multicolumn{8}{|l|}{ Follow-up ADC all ALS patients $(n=15)>$ controls } \\
\hline Precentral Gyrus, WM & r & 3258 & 24 & -20 & 63 & 6.18 & 0.036 \\
\hline Precentral Gyrus, subgyral, WM & r & & 12 & -21 & 62 & 5.01 & $0.001^{*}$ \\
\hline Precentral Gyrus, subgyral, WM, Corona radiata & r & & 18 & -20 & 43 & 4.1 & $0.013^{*}$ \\
\hline Sub-lobar, Extra-Nuclear, WM, Internal capsule, Crus post. & r & 379 & 18 & -13 & -1 & 4.32 & $0.008^{*}$ \\
\hline Sub-lobar, Extra-Nuclear, WM, Internal capsule, Crus post. & I & 1234 & -18 & -13 & -2 & 4.28 & $0.009^{*}$ \\
\hline Sub-lobar, Extra-Nuclear, WM, Internal capsule, Crus post. & 1 & & -19 & -12 & 6 & 4.13 & $0.012^{*}$ \\
\hline Precentral Gyrus, WM & 1 & 351 & -9 & -25 & 65 & 4.27 & $0.009^{*}$ \\
\hline Precentral Gyrus, subgyral, WM, Corona radiata & 1 & 1148 & -37 & -11 & 43 & 4.16 & $0.011^{*}$ \\
\hline Precentral Gyrus, subgyral, WM, Corona radiata & 1 & & -28 & -25 & 50 & 4.16 & $0.012^{*}$ \\
\hline Precentral Gyrus, subgyral, WM, Corona radiata & 1 & & -25 & -22 & 42 & 4.06 & $0.012^{*}$ \\
\hline subgyral, WM, Corona radiata, below BA 6 & 1 & 241 & -13 & -14 & 59 & 4.08 & $0.014^{*}$ \\
\hline
\end{tabular}

by means of the paired t-test. FA was significantly reduced at follow-up in the precentral gyrus, CST (at the mesencephal level), in the cerebellum (culmen, declive), the temporal and parietal lobe. Diffusivity was significantly enhanced after 6 months both in the internal and external capsule (Table 3, Figure 2).

\section{Correlation with clinical scores at T0}

Disease duration negatively correlated with FA in precentral gyrus, CST (corona radiate, internal capsule) and the cerebellum, which means that in these areas FA decreases with time course of ALS. Changes in diffusivity did not correlate with disease duration. The ALSFRS-R positively correlated with FA in the CST, and the cerebellum. The ALSFRS-R did not correlate with diffusivity. Physical and executive function, reflected in SF36 subscore, positively correlated with FA in the cerebellum, but did not correlate with changes in diffusivity. This means that worsening ALS, indicated by a decrease of ALSFRS-R or SF36 subscore, is reflected as FA decrease in the CST and cerebellum (Table 4).

\section{Correlation with clinical scores at follow-up}

After 6 months, the DTI data were correlated with clinical parameters. With respect to SVC, the FA negatively correlated with disease duration along the CST in the internal and external capsule. However, the decrease of FA was higher on the left side. Again diffusivity changes did not correlate with disease duration. ALSFRS-R positively correlated with FA in the precentral gyrus, the CST (corona radiate), the WM of right middle frontal gyrus and supplementary motor cortex. ALSFRS-R did not correlate with diffusivity changes. Physical and executive function (SF36 subscore) positively correlated with FA in the CST at brain stem level and negatively with diffusivity in the cerebellum (anterior lobe, dentatus, fastigium) and parahippocampal gyrus (Table 4).

\section{Discussion}

Involvement of the white matter in ALS is increasingly being recognized and extensive white matter abnormalities can be found in the region of the central sulcus and the CST, extending across the corpus callosum and into 


\section{FA TO $n=24$}

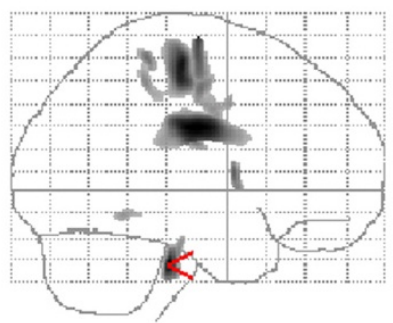

\section{FA follow-up $n=15$}

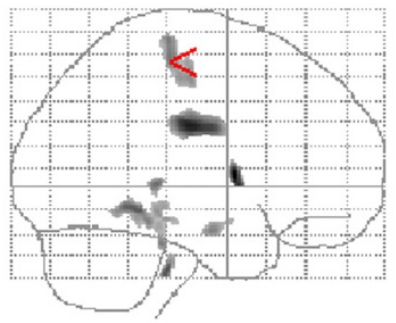

\section{ADC TO $n=24$}

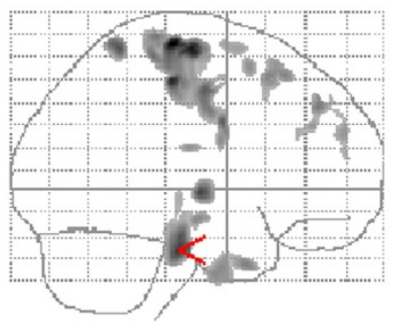

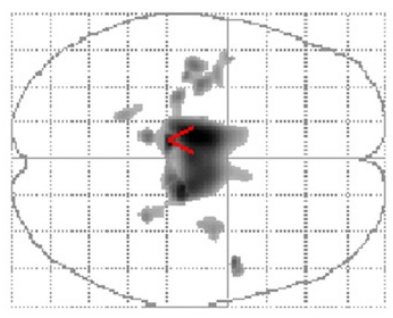
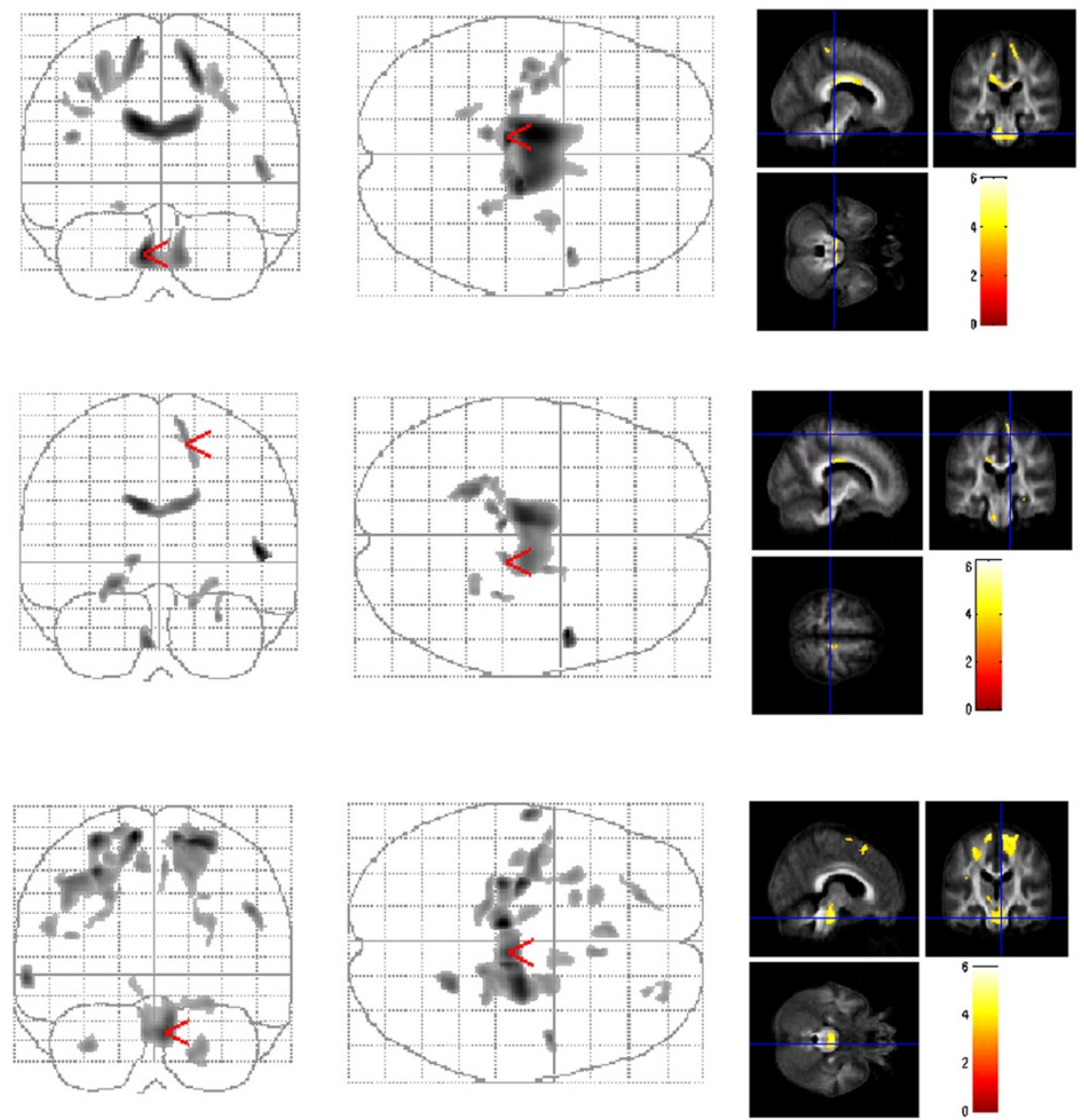

\section{ADC follow-up $n=15$}
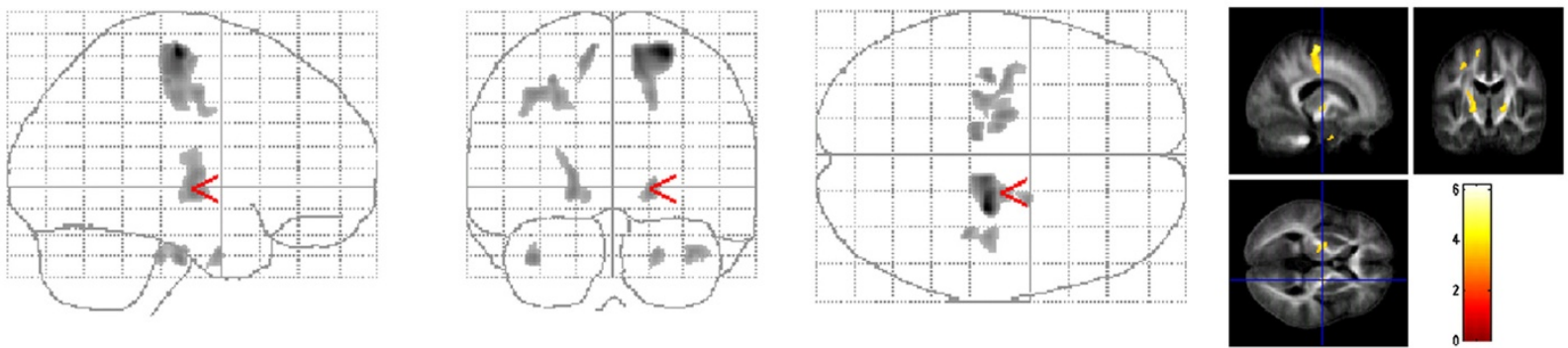

Figure 1 Group comparisons of ALS patients versus controls. A results are superimposed on the mean of all subjects of spatially normalized anisotropy images. Voxels with a significant decrease in FA in the patients versus controls are found in the precentral gyrus, the CST and pons ( $p<0,001$ uncorrected). ADC results are superimposed on the mean of all subjects of spatially normalized anisotropy images. Voxels with a significant increase in ADC in the patients versus controls are found in the precentral gyrus, CST, pons, frontal ares and limbic system ( $p<0,001$ uncorrected).

the frontal lobes [1]. The intact axonal membrane and the myelin coating of axons are important determinants for anisotropic diffusion in neural tissues [13] and therefore, a reduced FA along the CST in ALS patients reflects axonal fibre degeneration and myelin pallor [1].

In the present study, microstructural changes in the brains of ALS patients could be demonstrated as decreased FA and increased diffusivity mainly in the pyramidal motor system, frontotemporal areas, limbic system, and the cerebellum. Comparison of the follow-up group with healthy probands revealed a progressive decrease in FA and diffusivity increase along the CST after 6 months. Increasing fiber tract pathology during disease course could be verified by direct 
Table 3 Results of direct comparisons of ALS patients and correlation analysis

\begin{tabular}{|c|c|c|c|c|c|c|c|}
\hline & Side & Cluster size & $\mathbf{x}$ & y & z & $\mathrm{T}$ & $\mathrm{p}$ \\
\hline \multicolumn{8}{|l|}{ Paired t-test FA TO / Follow-up } \\
\hline Temporal Lobe, Superior Temporal Gyrus, GM, BA 39 & I & 879 & -49 & -62 & 28 & 7.5 & $0.001^{*}$ \\
\hline Temporal Lobe, Superior Temporal Gyrus, GM, BA 39 & I & & -56 & -64 & 16 & 4.82 & $0.033^{*}$ \\
\hline Parietal Lobe, Supramarginal Gyrus, GM, BA 40 & I & & -58 & -61 & 30 & 4.19 & $0.075^{*}$ \\
\hline Cerebellum, Posterior Lobe, Declive & r & 921 & 24 & -59 & -25 & 6.88 & $0.002^{*}$ \\
\hline Cerebellum, Anterior Lobe, Culmen & r & & 34 & -52 & -21 & 4.25 & $0.07^{*}$ \\
\hline Precentral Gyrus, WM & $r$ & 743 & 44 & -9 & 41 & 6.67 & $0.003^{*}$ \\
\hline Precentral Gyrus, WM & r & & 53 & -6 & 43 & 5.56 & $0.012^{*}$ \\
\hline Superior Temporal Gyrus, WM & $r$ & 2014 & 65 & -39 & 10 & 6.36 & $0.004^{*}$ \\
\hline Superior Temporal Gyrus, GM, BA 22 & $r$ & & 61 & -59 & 16 & 5.95 & $0.008^{*}$ \\
\hline Superior Temporal Gyrus, GM, BA 22 & r & & 65 & -48 & 20 & 5.12 & $0.022^{*}$ \\
\hline Parietal Lobe, Precuneus, WM & $r$ & 582 & 35 & -74 & 39 & 6.21 & $0.005^{*}$ \\
\hline Brainstem, Midbrain, CST & $r$ & 270 & 16 & -16 & -17 & 5.89 & $0.008^{*}$ \\
\hline Temporal Lobe & $r$ & 756 & 44 & 8 & -42 & 5.72 & $0.01^{*}$ \\
\hline Superior Frontal Gyrus, WM & r & 221 & 35 & 39 & 35 & 5.27 & $0.018^{*}$ \\
\hline Middle Frontal Gyrus, subgyral, WM & r & & 29 & 34 & 31 & 4.76 & $0.035^{*}$ \\
\hline Superior Frontal Gyrus, GM, BA 11 & r & 487 & 28 & 45 & -18 & 4.90 & $0.03^{*}$ \\
\hline Temporal Lobe, Middle Temporal Gyrus, WM & r & 253 & 60 & -7 & -9 & 4.77 & $0.035^{*}$ \\
\hline Temporal Lobe, Superior Temporal Gyrus, WM & $r$ & & 57 & -2 & 3 & 4.33 & $0.063^{*}$ \\
\hline \multicolumn{8}{|l|}{ Paired t-test ADC T0 / Follow-up } \\
\hline Extra-Nuclear, WM, External capsule & I & 2054 & -28 & 2 & -3 & 6.38 & $0.003^{*}$ \\
\hline Extra-Nuclear, WM, External capsule & 1 & & -29 & -15 & 11 & 6.16 & $0.004^{*}$ \\
\hline Extra-Nuclear, WM, External capsule & I & & -25 & -1 & 8 & 5.5 & $0.01^{*}$ \\
\hline Extra-Nuclear, WM, Internal capsule & I & 383 & -19 & 8 & 3 & 5.94 & $0.005^{*}$ \\
\hline
\end{tabular}

Ordered by descending T-values. R=right; $\mathrm{L}=\mathrm{left}$, ${ }^{*}$ SVC $10 \mathrm{~mm}$ (FWE).

comparison of T0 with the follow-up DTI data from 15 patients. Brain structure pathology correlated well with disease duration, ALSFRS-R andthe SF-36 subscore for physical and executive function, predominantly in the range of motor system, but interestingly also in the cerebellum and the limbic system.

\section{Widespread DTI changes in motoric and extramotoric systems in ALS}

The decreased FA in the internal capsule and premotor cortex is in accordance with several DTI studies in ALS [4,6,8,9,14-23]. Microstructural alterations in frontal areas are in line with bilateral frontal atrophy in VBM [11,24-26], an increase of diffusivity in frontal regions $[21,27]$ and observed in post mortem brain tissues [1] Since the corpus callosum connects orbitofrontal and frontal cortices, its involvement in ALS is a consistent feature $[9,28]$ and together with the known DTI changes in the hippocampal formations, cingulum, and frontal white matter, may reflect cognitive impairment, which is often recognized in patients with ALS [29,30]. However, aside from ruling out clinical significant dementia by means of MMSE and FAB, we did not undertake detailed neuropsychological testing.

\section{Involvement of cerebellum in ALS}

The cerebellum has been little studied in ALS, probably because of the lack of cerebellar signs in most patients. However, evidence for the involvement of cerebellum in ALS derives from early pathological [31,32] and functional magnetic resonance imaging (fMRI) studies. Besides the enhanced activity in sensorimotor network, fMRI studies in ALS have demonstrated the recruitment of additional areas, such as the cerebellum and basal ganglia [33-37]. Hence, it is probable that functional compensation in ALS relies on existing resources and mechanisms and not on development of new synapses or pathways [35]. Structural changes of cerebellum in ALS could be demonstrated as regional white matter alterations [26] and decreased grey matter volume [38] in VBM and as increased diffusivity in the anterior lobe of the cerebellum bilateral [21].

In the present study, the direct comparison after 6 months showed a reduced FA in the cerebellum (culmen, 


\section{FA t-test T0 vs. follow-up}
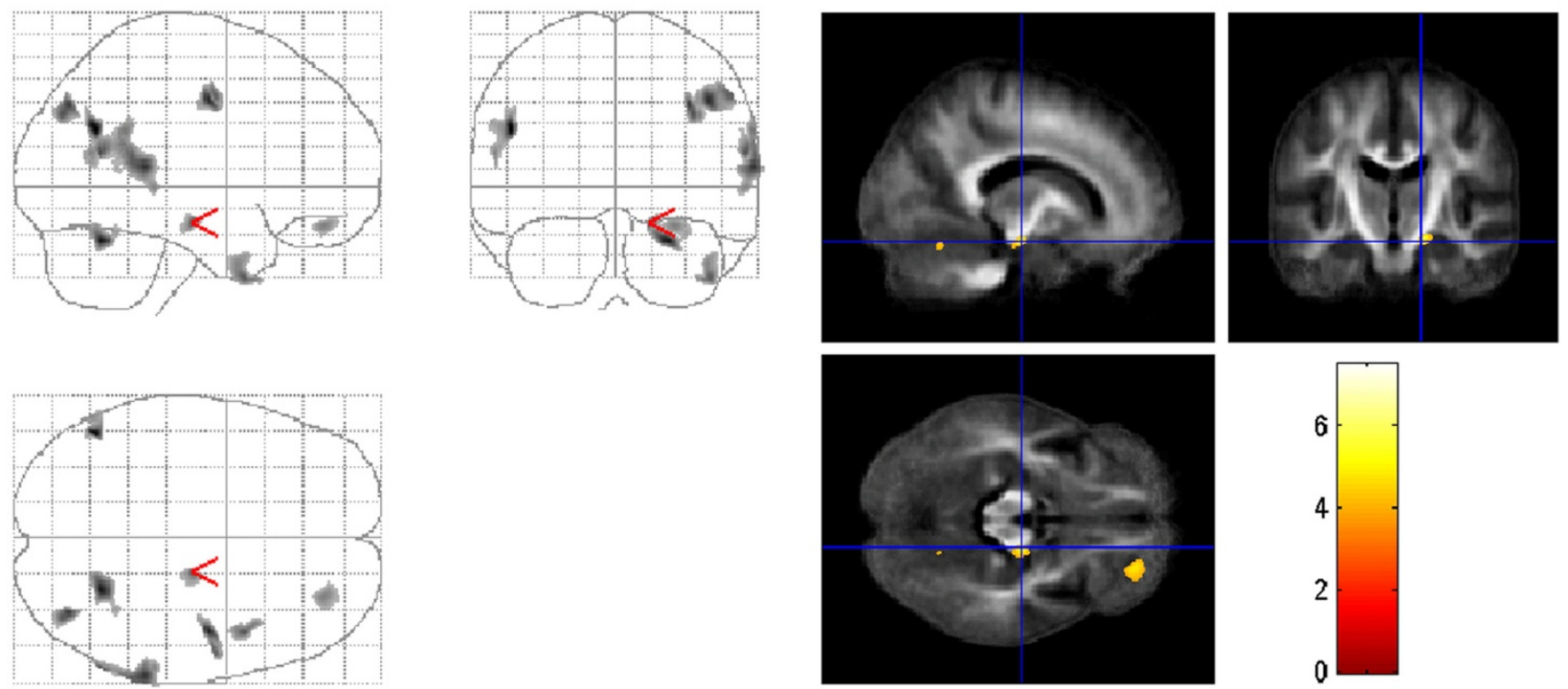

\section{ADC t-test T0 vs. follow-up}
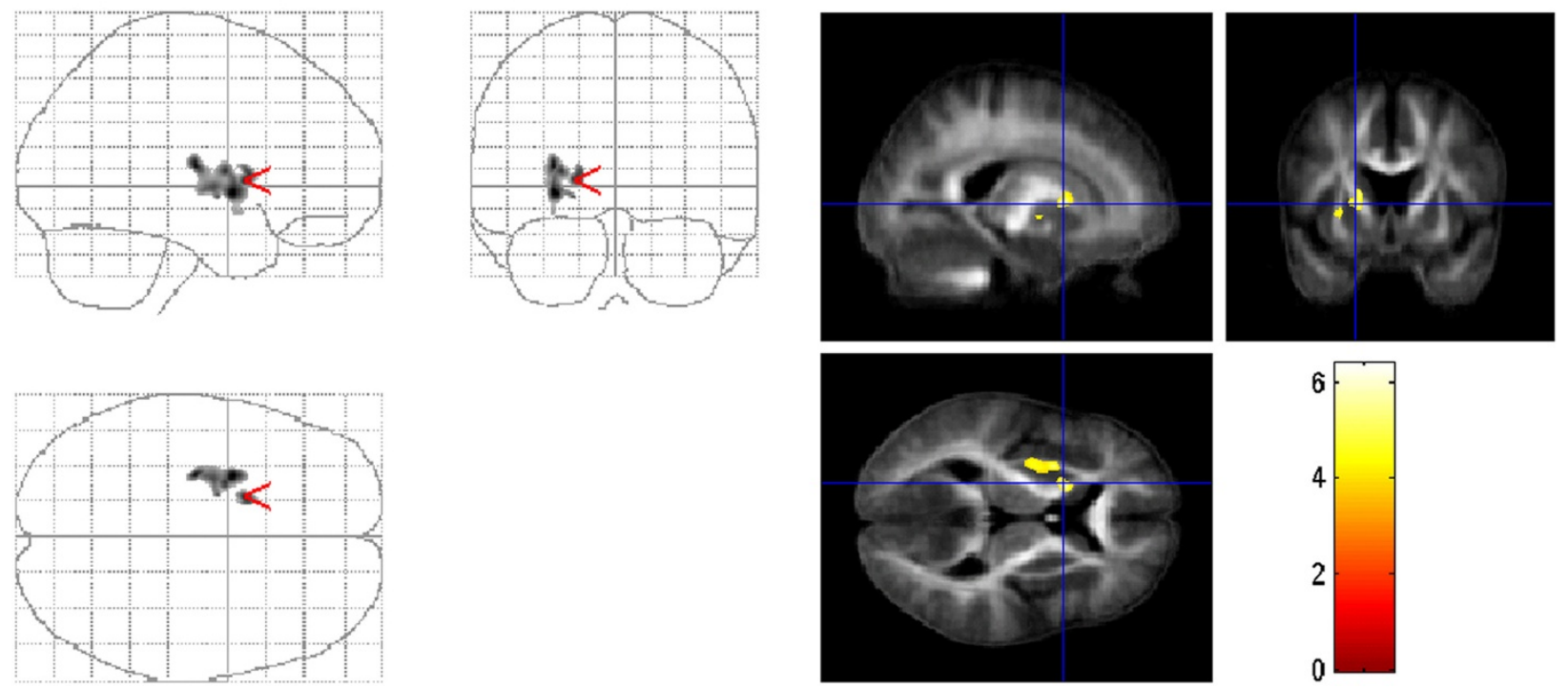

Figure 2 Direct comparison between T0 and follow-up in ALS patients. Comparison of FA results between T0 and follow-up ( $\mathrm{n}=15)$ with paired t-test superimposed on the mean of all subjects of spatially normalized anisotropy images. FA is significantly reduced in the follow-up in the precentral gyrus, CST (at the mesencephal level), in the cerebellum (culmen, declive), the temporal and parietal lobe ( $p<0,001$ uncorrected). Comparison of ADC results between T0 and follow-up $(n=15)$ with paired t-test superimposed on the mean of all subjects of spatially normalized anisotropy images. Diffusivity is significantly enhanced after 6 months in the internal and external capsule ( $p<0,001$ uncorrected).

declive) of ALS patients. These structural changes correlated with disease duration, ALSFRS-R, physical and executive functions. Since, the cerebellum contributes to coordination and improvement of motor performance and is involved in motor learning [39], the abnormal connectivity found between the supplementary motor cortex and cerebellar areas is therefore not only of functional, but also of a structural nature [35,37]. One could hypothesise that as a consequence of limited resources in the motor cortex, these cerebellar regions are activated due to an enhanced modulatory response in ALS. However, this compensation is paralleled by a progressive structural alteration of connectivity, finally leading to an ineffective modulation. However, the exact clinical and pathological relevance of these findings is not quite clear. We speculate that the structural changes in the 
Table 4 Correlations with clinical data

\begin{tabular}{|c|c|c|c|c|c|c|c|}
\hline & Side & Cluster size & $\mathbf{x}$ & y & z & $\mathbf{T}$ & $\mathbf{p}$ \\
\hline \multicolumn{8}{|l|}{ T0 FA correlation with ALSFRS-R } \\
\hline Right Brainstem, Midbrain & r & 233 & 10 & -24 & -21 & 4.41 & $0.019^{*}$ \\
\hline Cerebellum, Posterior Lobe, Declive & r & 251 & 13 & -58 & -19 & 3.99 & $0.04^{*}$ \\
\hline \multicolumn{8}{|l|}{ T0 FA correlation with disease duration } \\
\hline Precentral Gyrus, WM, below BA 6 & $r$ & 596 & 4 & -27 & 70 & 4.93 & $0.007^{*}$ \\
\hline Precentral Gyrus, subgyral, WM, Corona radiata & r & & 6 & -30 & 61 & 4.52 & $0.014^{*}$ \\
\hline Sub-lobar, WM, CST, Internal capsule & 1 & 867 & -31 & -26 & 17 & 4.58 & $0.013^{*}$ \\
\hline Sub-lobar, WM, CST, Internal capsule & I & & -30 & -18 & 15 & 4.47 & $0.017^{*}$ \\
\hline Cerebellum, Anterior Lobe, Culmen & $r$ & 317 & 12 & -56 & -15 & 4.56 & $0.014^{*}$ \\
\hline Sub-lobar, WM, CST, Corona radiata & I & 202 & -13 & -29 & 51 & 4.37 & $0.02^{*}$ \\
\hline \multicolumn{8}{|l|}{ T0 FA correlation with SF36 } \\
\hline Cerebellum, Anterior Lobe, Culmen & $r$ & 715 & 19 & -43 & -27 & 5.24 & $0.004^{*}$ \\
\hline Cerebellum, Anterior Lobe & $r$ & & 10 & -42 & -33 & 4.4 & $0.019^{*}$ \\
\hline Cerebellum, Anterior Lobe & I & 561 & -10 & -41 & -33 & 4.84 & $0.008^{*}$ \\
\hline Cerebellum, Anterior Lobe, Culmen & 1 & & -16 & -42 & -27 & 4.22 & $0.027^{*}$ \\
\hline \multicolumn{8}{|l|}{ Follow-up FA correlation with ALSFRS-R } \\
\hline Precentral Gyrus, subgyral, WM, BA 6 & $r$ & 469 & 50 & 2 & 38 & 6.35 & $0.006^{*}$ \\
\hline Precentral Gyrus, subgyral, WM, BA 6 & $r$ & & 46 & -5 & 46 & 6.11 & $0.008^{*}$ \\
\hline Frontal Lobe, Middle Frontal Gyrus, WM & r & 233 & 33 & 13 & 50 & 5.99 & $0.01^{*}$ \\
\hline Precentral Gyrus, WM, Corona radiata & r & 417 & 29 & -15 & 56 & 5.87 & $0.011^{*}$ \\
\hline Precentral Gyrus, WM, Corona radiata & I & 219 & -16 & -12 & 59 & 5.01 & $0.014^{*}$ \\
\hline \multicolumn{8}{|l|}{ Follow-up FA correlation with disease duration } \\
\hline Capsula Inerna, Crus post., CST & 1 & 1722 & -24 & -19 & 2 & 6.87 & $0.003^{*}$ \\
\hline Sub-lobar, WM, Capsula Inerna, Crus post., CST & 1 & & -28 & -25 & 14 & 5.7 & \\
\hline Internal capsule, Crus post., CST & r & 408 & 24 & -16 & -4 & 5.69 & $0.014^{*}$ \\
\hline Internal capsule, Crus post., CST & r & & 32 & -17 & -5 & 4.12 & \\
\hline External capsule & r & 431 & 31 & -12 & 10 & 5.61 & $0.015^{*}$ \\
\hline \multicolumn{8}{|l|}{ Follow-up FA correlation with SF36 } \\
\hline Brainstem, Pons, CST & I & 387 & -11 & -32 & -34 & 5.37 & $0.02^{*}$ \\
\hline
\end{tabular}

cerebellum observed in the current study reflect a compensatory mechanism for the progressive loss of motor function. Further studies should evaluate cerebellar signs in more detail and undertake a comparison of cerebellar involvment in familal and sporadic ALS. Nevertheless with regard to studying cerebellar structures using DTI, several methodological limitations have to be taken into account such as the low resolution after normalization and the incomplete mapping of cerebellum. Moreover, measurement of radial and axial diffusivities would provide more specific information relating to diffusion tensor.

\section{Follow-up}

The few existing longitudinal DTI studies have captured the progression of upper motor neurone degeneration in ALS by demonstrating decreasing FA over time along the CST [40-42]. Fibertracking of the mean CST showed a reduction of FA in a follow-up examination of ALS patients, indicating that degeneration of the CST appears to worsen over time [7]. In another six-month follow-up study, ALS patients showed DTI abnormalities that extended mainly into the bilateral frontal lobes [43]. Accordingly in our study, the disease progression was reflected in a progressive FA decrease along the CST, which correlated with disease duration and the decrease of ALSFRS-R. However, it is important to note that ALSFRS-R is a composite score of upper and lower motor neuron components, whereas FA changes along the CST may reflect more upon upper motor neuron degeneration. For further studies, it would be useful to correlate FA changes with ALSFRS-R subscores.

Furthermore, it remains unclear as to why regions with FA decrease (corona radiate, corpus callosum, brain stem) after 6 months appear smaller. In addition to method-based explanations, a possible interaction with 
swollen neighbouring motor neurones or astrocytes is also a plausible reason that could be discussed. Finally, we detected at 6 months follow-up that increase in diffusivity progressed in a caudal (CST) and frontal direction (premotor cortex). The direct comparison of follow-up patients with T0 data supports the observed progression of FA changes and revealed microstructural changes in regions which were not detected when comparing ALS patients with normal controls in the group comparison.

\section{Conclusions}

In summary, our study provides further evidence for the well known structural changes in the CST and in frontal areas in the brains of ALS. Further, we demonstrate the involvement of cerebellum as a feature of disturbed connectivity in ALS, supported by the correlation analysis with clinical datasets. It seems that the extent of ALSrelated DTI abnormalities is greater than those identified by VBM analysis $[9,21]$, and that DTI can serve as stable biomarker in ALS [44].

\section{Abbreviations}

ADC: Apparent diffusion coefficient; ALS: Amyotrophic lateral sclerosis; CST: Corticospinal tract; DTI: Diffusion tensor imaging; FA: Fractional anisotropy.

\section{Competing interests}

Carsten Keil reports no disclosures. Tino Prell reports no disclosures. Thomas Peschel reports no disclosures. Viktor Hartung reports no disclosures. Reinhard Dengler reports no disclosures with regard to the current study. RD received honoraria, research grants, and travel grants from PharmAllergan, Ipsen Pharma, Merz Pharma, Boehringer-Ingelheim and Bayer Health Care. Julian Grosskreutz reports no disclosures.

\section{Authors' contributions}

Conceived and designed the experiments: JG, TP, CK, RD. Acquisition of data: CK. Analyzed the data: CK, JG, ThP. Wrote the paper: TP. All authors read and approved the final manuscript.

\section{Acknowledgement}

We thank Nasim Kroegel for proofreading.

\section{Author details}

'Department of Neurology, Jena University Hospital, Erlanger Allee 101, Jena 07747, Germany. ${ }^{2}$ Department of Psychiatry and Psychotherapy, Medical School Hannover, Carl-Neuberg-Strasse 1, Hannover 30625, Germany. ${ }^{3}$ Department of Neurology and Clinical Neurophysiology, Medical School Hannover, Carl-Neuberg-Str.1, Hannover 30625, Germany.

Received: 12 May 2012 Accepted: 31 October 2012

Published: 8 November 2012

\section{References}

1. Smith MC: Nerve fibre degeneration in the brain in amyotrophic lateral sclerosis. J Neurol Neurosurg Psychiatry 1960, 23:269-282.

2. Rafalowska J, Dziewulska D: White matter injury in amyotrophic lateral sclerosis (ALS). Folia Neuropathol 1996, 34:87-91.

3. Grosskreutz J, Peschel T, Unrath A, Dengler R, Ludolph AC, Kassubek J: Whole brain-based computerized neuroimaging in ALS and other motor neuron disorders. Amyotroph Lateral Scler 2008, 9:238-248.

4. Sach M, Winkler G, Glauche V, Liepert J, Heimbach B, Koch MA, et al: Diffusion tensor MRI of early upper motor neuron involvement in amyotrophic lateral sclerosis. Brain 2004, 127:340-350.
5. Abe O, Yamada H, Masutani Y, Aoki S, Kunimatsu A, Yamasue $H$, et al: Amyotrophic lateral sclerosis: diffusion tensor tractography and voxel-based analysis. NMR Biomed 2004, 17:411-416.

6. Agosta F, Pagani E, Rocca MA, Caputo D, Perini M, Salvi F, et al: Voxel-based morphometry study of brain volumetry and diffusivity in amyotrophic lateral sclerosis patients with mild disability. Hum Brain Mapp 2007, 28:1430-1438.

7. Sage CA, Peeters RR, Gorner A, Robberecht W, Sunaert S: Quantitative diffusion tensor imaging in amyotrophic lateral sclerosis. Neuroimage 2007, 34:486-499.

8. Muller HP, Lule D, Unrath A, Ludolph AC, Riecker A, Kassubek J: Complementary image analysis of diffusion tensor imaging and 3-dimensional t1-weighted imaging: white matter analysis in amyotrophic lateral sclerosis. J Neuroimaging 2011, 21:24-33.

9. Douaud G, Filippini N, Knight S, Talbot K, Turner MR: Integration of structural and functional magnetic resonance imaging in amyotrophic lateral sclerosis. Brain 2011, 134(Pt 12):3470-3479.

10. Grosskreutz J, Kaufmann J, Fradrich J, Dengler R, Heinze HJ, Peschel T: Widespread sensorimotor and frontal cortical atrophy in Amyotrophic Lateral Sclerosis. BMC Neurol 2006, 6:17.

11. Grosskreutz J, Kaufmann J, Fraedrich J, Dengler R, Heinze HJ, Peschel T: Widespread sensorimotor and frontal cortical atrophy in Amyotrophic Lateral Sclerosis. BMC Neurol 2006, 6:17.

12. Turner MR, Cagnin A, Turkheimer FE, Miller CC, Shaw CE, Brooks DJ, et al: Evidence of widespread cerebral microglial activation in amyotrophic lateral sclerosis: an [11C](R)-PK11195 positron emission tomography study. Neurobiol Dis 2004, 15:601-609.

13. Beaulieu C: The basis of anisotropic water diffusion in the nervous system - a technical review. NMR Biomed 2002, 15:435-455.

14. Ellis CM, Simmons A, Jones DK, Bland J, Dawson JM, Horsfield MA, et al: Diffusion tensor MRI assesses corticospinal tract damage in ALS. Neurology 1999, 53:1051-1058.

15. Graham JM, Papadakis N, Evans J, Widjaja E, Romanowski CA, Paley MN, et al: Diffusion tensor imaging for the assessment of upper motor neuron integrity in ALS. Neurology 2004, 63:2111-2119.

16. Iwata NK, Aoki S, Okabe S, Arai N, Terao Y, Kwak S, et al: Evaluation of corticospinal tracts in ALS with diffusion tensor MRI and brainstem stimulation. Neurology 2008, 70:528-532.

17. Yin H, Cheng SHT, Zhang J, Ma L, Gao Y, Li D, et al: Corticospinal tract degeneration in amyotrophic lateral sclerosis: a diffusion tensor imaging and fibre tractography study. Ann Acad Med Singapore 2008, 37:411-415.

18. Lombardo F, Frijia F, Bongioanni P, Canapicchi R, Minichilli F, Bianchi F, et al: Diffusion tensor MRI and MR spectroscopy in long lasting upper motor neuron involvement in amyotrophic lateral sclerosis. Arch Ital Biol 2009, 147:69-82.

19. Sage CA, Van Hecke W, Peeters R, Sijbers J, Robberecht W, Parizel P, et al: Quantitative diffusion tensor imaging in amyotrophic lateral sclerosis: revisited. Hum Brain Mapp 2009, 30:3657-3675.

20. Senda J, Ito M, Watanabe $H$, Atsuta N, Kawai $Y$, Katsuno M, et al: Correlation between pyramidal tract degeneration and widespread white matter involvement in amyotrophic lateral sclerosis: A study with tractography and diffusion-tensor imaging. Amyotroph Lateral Scler 2009, 10(5-6):288-294

21. Canu E, Agosta F, Riva N, Sala S, Prelle A, Caputo D, et al: The topography of brain microstructural damage in amyotrophic lateral sclerosis assessed using diffusion tensor MR imaging. AJNR Am J Neuroradiol 2011, 32:1307-1314

22. Iwata NK, Kwan JY, Danielian LE, Butman JA, Tovar-Moll F, Bayat E, et al: White matter alterations differ in primary lateral sclerosis and amyotrophic lateral sclerosis. Brain 2011, 134:2642-2655.

23. Li J, Pan P, Song W, Huang R, Chen K, Shang H: A meta-analysis of diffusion tensor imaging studies in amyotrophic lateral sclerosis. Neurobiol.Aging 2011, 33(8):1833-1888.

24. Ellis CM, Suckling J, Amaro E Jr, Bullmore ET, Simmons A, Williams SC, et at: Volumetric analysis reveals corticospinal tract degeneration and extramotor involvement in ALS. Neurology 2001, 57:1571-1578.

25. Chang JL, Lomen-Hoerth C, Murphy J, Henry RG, Kramer JH, Miller BL, et al A voxel-based morphometry study of patterns of brain atrophy in ALS and ALS/FTLD. Neurology 2005, 65:75-80.

26. Kassubek J, Unrath A, Huppertz HJ, Lule D, Ethofer T, Sperfeld AD, et al: Global brain atrophy and corticospinal tract alterations in ALS, as 
investigated by voxel-based morphometry of 3-D MRI. Amyotroph Lateral Scler Other Motor Neuron Disord 2005, 6:213-220.

27. Ciccarelli O, Behrens TE, Johansen-Berg H, Talbot K, Orrell RW, Howard RS, et al: Investigation of white matter pathology in ALS and PLS using tract-based spatial statistics. Hum Brain Mapp 2009, 30:615-624.

28. Filippini N, Douaud G, Mackay CE, Knight S, Talbot K, Turner MR: Corpus callosum involvement is a consistent feature of amyotrophic lateral sclerosis. Neurology 2010, 75:1645-1652.

29. Abrahams S, Leigh PN, Goldstein LH: Cognitive change in ALS: a prospective study. Neurology 2005, 64:1222-1226.

30. Raaphorst J, de Visser M, van Tol MJ, Linssen WH, van der Kooi AJ, de Haan $\mathrm{RJ}$, et al: Cognitive dysfunction in lower motor neuron disease: executive and memory deficits in progressive muscular atrophy. I Neurol Neurosurg Psychiatry 2011, 82:170-175.

31. Swash M, Scholtz CL, Vowles G, Ingram DA: Selective and asymmetric vulnerability of corticospinal and spinocerebellar tracts in motor neuron disease. J Neurol Neurosurg Psychiatry 1988, 51:785-789.

32. Takahashi H, Oyanagi K, Ikuta F, Tanaka M, Yuasa T, Miyatake T: Widespread multiple system degeneration in a patient with familial amyotrophic lateral sclerosis. J Neurol Sci 1993, 120:15-21.

33. Konrad C, Henningsen H, Bremer J, Mock B, Deppe M, Buchinger C, et al: Pattern of cortical reorganization in amyotrophic lateral sclerosis: a functional magnetic resonance imaging study. Exp Brain Res 2002, 143:51-56.

34. Konrad C, Jansen A, Henningsen H, Sommer J, Turski PA, Brooks BR, et al: Subcortical reorganization in amyotrophic lateral sclerosis. Exp Brain Res 2006, 172:361-369.

35. Schoenfeld MA, Tempelmann C, Gaul C, Kuhnel GR, Duzel E, Hopf JM, et al: Functional motor compensation in amyotrophic lateral sclerosis. J Neurol 2005, 252:944-952

36. Han J, Ma L: Functional magnetic resonance imaging study of the brain in patients with amyotrophic lateral sclerosis. Chin Med Sci J 2006, 21:228-233.

37. Agosta F, Valsasina P, Absinta M, Riva N, Sala S, Prelle A, et al: Sensorimotor functional connectivity changes in amyotrophic lateral sclerosis. Cereb Cortex 2011, 21:2291-2298.

38. Thivard L, Pradat PF, Lehericy S, Lacomblez L, Dormont D, Chiras J, et al: Diffusion tensor imaging and voxel based morphometry study in amyotrophic lateral sclerosis: relationships with motor disability. J Neurol Neurosurg Psychiatry 2007, 78:889-892.

39. Penhune VB, Doyon J: Cerebellum and M1 interaction during early learning of timed motor sequences. Neuroimage 2005, 26:801-812

40. Jacob S, Finsterbusch J, Weishaupt JH, Khorram-Sefat D, Frahm J, Ehrenreich $H$ : Diffusion tensor imaging for long-term follow-up of corticospinal tract degeneration in amyotrophic lateral sclerosis. Neuroradiology 2003, 45:598-600.

41. Nickerson JP, Koski CJ, Boyer AC, Burbank HN, Tandan R, Filippi CG: Linear longitudinal decline in fractional anisotropy in patients with amyotrophic lateral sclerosis: preliminary results. Klin Neuroradio/ 2009, 19:129-134

42. Zhang Y, Schuff N, Woolley SC, Chiang GC, Boreta L, Laxamana J, et al: Progression of white matter degeneration in amyotrophic lateral sclerosis: A diffusion tensor imaging study. Amyotroph Lateral Scler 2011, 12:421-429.

43. Senda J, Kato S, Kaga T, Ito M, Atsuta N, Nakamura T, et al: Progressive and widespread brain damage in ALS: MRI voxel-based morphometry and diffusion tensor imaging study. Amyotroph Lateral Scler 2011, 12:59-69.

44. Turner MR, Grosskreutz J, Kassubek J, Abrahams S, Agosta F, Benatar M, et al: Towards a neuroimaging biomarker for amyotrophic lateral sclerosis. Lancet Neurol 2011, 10:400-403.

doi:10.1186/1471-2202-13-141

Cite this article as: Keil et al: Longitudinal diffusion tensor imaging in amyotrophic lateral sclerosis. BMC Neuroscience 2012 13:141.

\section{Submit your next manuscript to BioMed Central and take full advantage of:}

- Convenient online submission

- Thorough peer review

- No space constraints or color figure charges

- Immediate publication on acceptance

- Inclusion in PubMed, CAS, Scopus and Google Scholar

- Research which is freely available for redistribution

Submit your manuscript at www.biomedcentral.com/submit
Biomed Central 